\begin{tabular}{|c|c|}
\hline Title & Characteristic Time, Length and V elocity Scales of Transverse Flows in the Surf Zone \\
\hline Author(s) & Otsuka, Junichi; W atanabe, Y asunori \\
\hline Citation & $\begin{array}{l}\text { Coastal engineering journal, } 57(2), 1550006 \\
\text { https://doi.org/10.1142/\$0578563415500060 }\end{array}$ \\
\hline Issue Date & 2015-06-08 \\
\hline Doc URL & http://hdl .handle.net/2115/62094 \\
\hline Rights & $\begin{array}{l}\text { Electronic version of an article published as Coastal engineering journal, Volume 57, Issue 2, 2015, } 1550006 \\
\text { DOl:10.1142/S0578563415500060 @ copyright W orld Scientific Publishing Company } \\
\text { http:/www.worldscientific.com/worldscinet/kej }\end{array}$ \\
\hline Type & article (author version) \\
\hline File Information & Characteristic time, length and velocity scales of transverse flows in the surf zone.pdf \\
\hline
\end{tabular}

Instructions for use 
July 29, 2015 12:11 WSPC/INSTRUCTION FILE "Characteristic time, length and velocity scales of transverse flows in the surf zone"

Coastal Engineering Journal

(c) World Scientific Publishing Company and Japan Society of Civil Engineers

\title{
Characteristic time, length and velocity scales of transverse flows in the surf zone
}

\author{
Junichi Otsuka \\ Civil engineering research institute for cold region \\ Hiragishi 1-3, Sapporo, 062 8602, Japan \\ 08900@ceri.go.jp \\ Yasunori Watanabe \\ School of Engineering, Hokkaido University \\ North 13 West 8, Sapporo, 060 8628, Japan \\ yasunori@eng.hokudai.ac.jp \\ Received (16 December 2014) \\ Revised (27 March 2015)
}

\begin{abstract}
An ultrasonic velocity profiler (UVP) acquires instantaneous distributions of axial velocity along the ultrasonic beam emitted from the transducer. In this paper, organizations of the transverse flows over a span of a laboratory wave flume in the surf zone were identified experimentally on the basis of UVP measurements. Ensemble mean divergent and convergent flows were observed over the span of the flume in the surf zone regardless of breaker conditions. By analogy with the flow evolution in a previous computation, by Watanabe and Saeki (1999), we identified that the organization of the wave-breaking-induced counterrotating vortices determined the spatial and temporal variations in the transverse flows at the early stages of the breaking process regardless of the widths of wave flumes and friction on the sidewalls and bottom. The observed transverse flows were driven by pairs of counter-rotating vortices produced under the breaking waves and the change in the orientation during the passage of breaking waves. The fundamental features of the transverse flows evolving in the breaking process, depending on the breaker type, are discussed statistically to parameterize velocity, time, and length scales of the variations of the transverse velocity. The dimensionless length and time scales had negative linear correlations with the Froude number (defined by relative transverse convection with respect to gravity) as well as a surf similarity parameter, which indicates that the gravity effect associated with the projections of the breaking wave crests defines the primary roller vorticity and shear intensity at the plunging location, resulting in destabilization of the flows in the transverse direction to form organized transverse flow structures.
\end{abstract}

Keywords: wave breaking, ultrasonic velocity profiler, transverse flow, vortex structure

\section{Introduction}

In a surf zone, rotational flows with turbulence, produced by breaking waves, evolve during the breaking process depending on the breaker type, typically plunging and spilling breakers. In the former, the overturning wave crest splashes on the forward 
July 29, 2015 12:11 WSPC/INSTRUCTION FILE "Characteristic time, length and velocity scales of transverse flows in the surf zone"

wave trough to produce secondary jets following energetic vortices in a transition region (or unsaturated region) before the splash-up behaviors culminate into boreshaped waves containing turbulence in a bore region (or saturated region). In the latter, the crest spilling down on the front face of the wave disturbs the surface flows, evolving into small-scale turbulence. Experimental and computational investigations have been performed to identify the cross-shore distributions of the surf-zone turbulent flows in horizontal-vertical two-dimensional space assuming uniform statistical features of turbulent motions in the direction of the wave crest (for review, see Longo 2002). However, uneven transverse fluid motions have also been observed in organized three-dimensional vortices produced after wave breaking, resulting in modifications of the local convection and diffusion in the surf zone (Nadaoka et al. 1989, Watanabe and Saeki 1999, Kubo and Sunamura 2001). Nadaoka et al. (1989) found three-dimensional turbulent structures involving vortex pairs extending in the direction obliquely downwards, a so-called obliquely descending eddy (ODE), behind the breaking wave face. Cox and Anderson (2001) observed the presence of vortical flows with a vertical axis, involved in ODE, using particle imaging velocimetry (PIV) measurements. Identical organizations of the flows configuring the transverse array of counter-rotating vortices have been found computationally by Christensen and Deigaard (2001) and Watanabe and Saeki (1999). Watanabe et al. (2005) found shear instability, manifested as stagnation point flow, emerging at wave-plunging locations, and triggers to amplify unstable undulations of the span-wise vorticity of the horizontal roller vortex. The resulting counter-rotating vortices are stretched obliquely downwards, and thus intensify to evolve into ODEs. The multiple pairs of sub-surface longitudinal vortices formed behind the breaking-wave front induce regular patterns of divergent and convergent surface flows above the vortices. This was identified experimentally on the basis of infrared measurements by Watanabe and Mori (2008). Kubo and Sunamura (2001) found another type of vertical flow experimentally, the so-called down-burst, also following an inherent three-dimensional velocity organization, which has been supported experimentally by PIV measurements providing vertical rotating fluid motions during the down-burst event (Ting 2006, 2008).

We performed high-resolution measurements of the transverse velocity in breaking waves using an ultrasonic velocity profiler (UVP). The UVP was developed for measuring instantaneous velocity profiles along an acoustic beam emitted from the transducer. The reliability and applicability of the UVP method have been examined for a variety of flow types (see Takeda, 1995). In coastal engineering, Longo et al. (2012) used the UVP to measure the mean vertical velocity and turbulence intensity under wind-generated water waves. The UVP method is advantageous in obtaining continuous time-dependent velocity profiles with relatively short sampling periods.

In this study, the transverse velocity distributions over the span of a wave flume for spilling and plunging waves were measured using UVP with the goal of under- 
July 29, 2015 12:11 WSPC/INSTRUCTION FILE "Characteristic time, length and velocity scales of transverse flows in the surf zone"

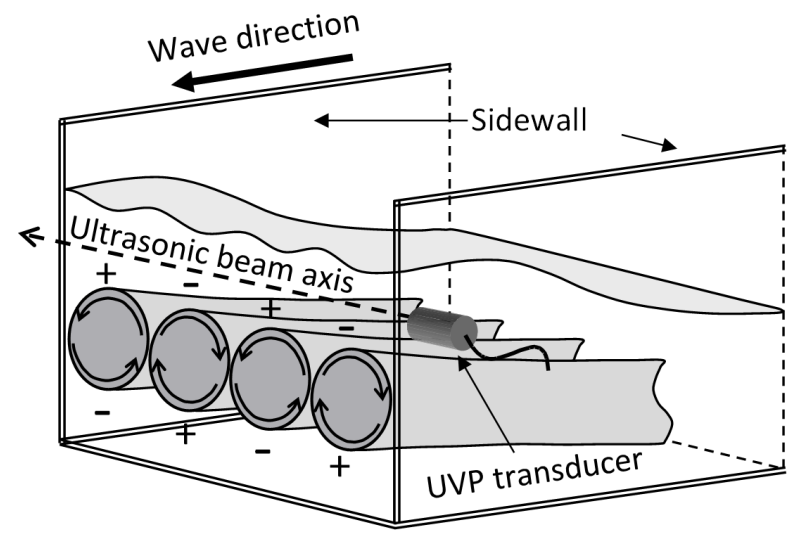

Fig. 1. Schematic illustration of positive and negative velocity patterns detected along the UVP measurement line above pairs of counter-rotating vortices.

standing the underlying features of the transverse organizations of the flows and the mechanical contributions to convection and diffusion processes in the surf zone. In particular, the measurements were motivated by a desire to obtain experimental evidence for the presence of counter-rotating vortex pairs or ODEs in the surf zone because UVP can detect the orientation of the instantaneous flows where organized vortex structures are formed (see Fig. 1). The transitions of length, time, and velocity scales characterizing the transverse flows involved in the vortex structures passing along the measurement axis were investigated statistically. In this study, we also discuss the governing factors determining these scales, and, in particular, dependence on the breaker type. In laboratory experiments, because the evolution of the transverse flows may be influenced by the sidewalls of the flume, the transverse velocities measured in two wave flumes with different widths were compared to examine the effects of the walls in the generation of the vortices.

This paper is organized as follows. The experimental set-up and conditions are explained in Section 2. In Section 3, the observed results associated with the transverse flows are explained, and the statistical features of the flow scales are discussed. The results are summarized in Section 4.

\section{Experimental set-up}

The laboratory experiments were performed in two wave flumes with different dimensions: A smaller flume (Flume 1) of 8.00-m length, 0.25-m width, and $0.60-\mathrm{m}$ depth with transparent acrylic walls (Fig. 2), and a larger flume (Flume 2) of a 24.0-m length, 0.6-m width and 1.0-m depth with transparent glass walls (see Fig. 3 ). Waves were generated with a piston-type wave generator at one end of each flume. In Flume 1, a hinge was installed on the bottom under the wave paddle, and at the other end of the flume, an oil jack was fixed at the bottom to create an 
July 29, 2015 12:11 WSPC/INSTRUCTION FILE "Characteristic time, length and velocity scales of transverse flows in the surf zone"

4 Junichi Otsuka and Yasunori Watanabe
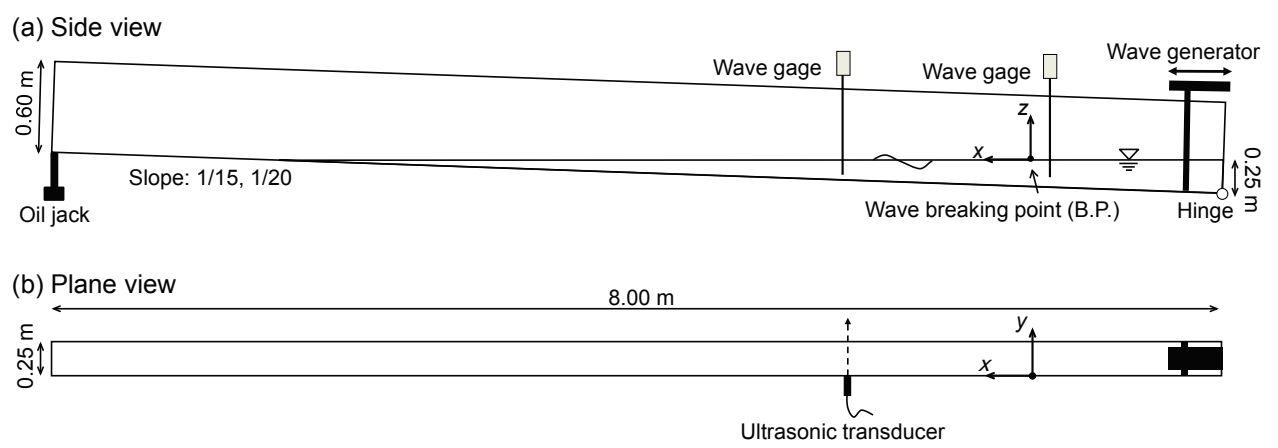

Fig. 2. Experimental set-up and coordinate system for flume 1.
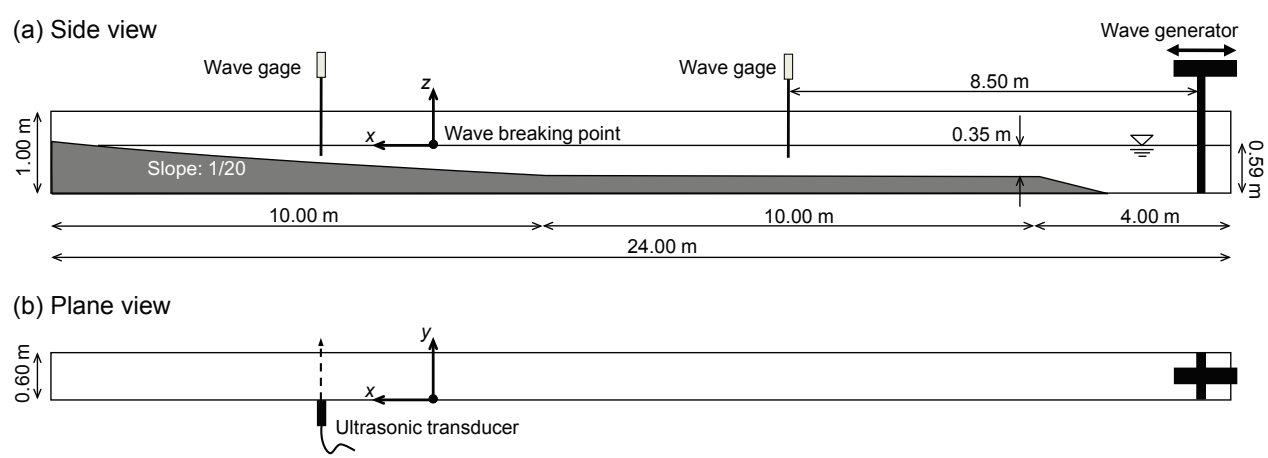

Fig. 3. Experimental set-up and coordinate system for flume 2 .

arbitrary beach slope by adjusting the jack. A 1:20 sloping concrete bed was fixed in Flume 2. $x^{-}, z_{-}$, and $y$-coordinates with the origin at the wave breaking point, still water level, and one side wall of the flume were defined to be the directions of wave propagation, vertically upward and perpendicular to the side wall, respectively (see Fig. 2 and Fig. 3). The experimental conditions for breaking waves and the bottom slope are listed in Table 1. Experiments for cases 1 through 7 were conducted in Flume 1, and that for case 8 in Flume 2, with a wider flume width than Flume 1, to examine the effects of the width to the flows through a comparison between case 7 and 8. Although there are minor differences in wave period and breaking wave height between cases 7 and 8 , because of the limitation of the available wave range for the wave generator, we confirmed the same breaking water depth, $h_{b}$, and surf similarity parameter, $\xi$, for both cases (see Table 1), indicating identical breakers between the cases. The dependency of the flow organization on breaker type was investigated for plunging and spilling waves on 1/15 and 1/20 sloping beds in Flume 1 . In case 7 , the cross-shore evolution of the flows was investigated at three locations near the breaking point $\left(x / h_{b}=2.3\right)$, in the transition region $\left(x / h_{b}=4.0\right)$ and in 
July 29, 2015 12:11 WSPC/INSTRUCTION FILE "Characteristic time, length and velocity scales of transverse flows in the surf zone"

the bore region $\left(x / h_{b}=8.5\right)$.

The UVP measurement was based on pulsed ultrasound echography. An ultrasound pulse at frequency $f_{0}(=2 \mathrm{MHz}$ in these experiments $)$ is emitted from a transducer along the ultrasonic beam axis, and the transducer receives the echo reflected from suspended micro particles mixed in the liquid flows. The fluid velocity $(v)$ in the direction of the emission is derived from the Doppler shift frequency $f_{D}$ by

$$
v=c f_{D} / 2 f_{0}
$$

where $c$ is the speed of sound in the liquid. The measurement position, from where the ultrasound is reflected on particles, along the beam axis is determined by

$$
y=c \delta / 2
$$

where $\delta$ is the time delay between the inception and reception time of the pulse burst.

The UVP transducer was attached on the outer face of the flume wall to emit ultrasonic pulses into the water across the wall to measure the transverse velocity distribution between the flume walls. The instantaneous velocity profiles were acquired at 128 points along the ultrasonic beam axis between the sidewalls of the flume at an acquisition frequency of $50 \mathrm{~Hz}$. Spatial resolutions of the velocity profiles were $2.0 \mathrm{~mm}$ for Flume 1 and $4.7 \mathrm{~mm}$ for Flume 2. The UVP was triggered by a transistor-transistor logic (TTL) signal that was emitted when a wave gauge, installed offshore from the sure zone, detected the first wave generated. Another wave gauge was placed at the same location as the velocity measurement and was synchronized with the UVP to activate simultaneously with the TTL signal. The synchronized velocity distributions and water surface elevations at the same location were described statistically on the basis of the ensemble average over 30 trials for each case.

In the highly aerated region, the measured velocity may be unreliable because entrained air bubbles interfere with the propagation of the ultrasonic pulses and cause erroneous burst signals. Thus, measurements were performed only in non-aerated regions under the wave trough level. Erroneous signals associated with dispersion from deeply entrained bubbles were reduced using a median digital filter. The UVP transducer was vertically traversed at $2.0-\mathrm{cm}$ intervals from the bottom to the wave trough.

It should be noted that distributions of all three velocity components are required to precisely identify the three-dimensional flow structures produced in the surf zone while the current experiments acquire only transverse velocity profiles representing transverse organizations of the flows. 
July 29, 2015 12:11 WSPC/INSTRUCTION FILE "Characteristic time, length and velocity scales of transverse flows in the surf zone"

6 Junichi Otsuka and Yasunori Watanabe

Table 1. Wave conditions

\begin{tabular}{|c|c|c|c|c|c|c|c|c|c|c|}
\hline Case & $\begin{array}{l}T \\
(\mathrm{~s})\end{array}$ & ${ }^{* 1} \begin{array}{l}H_{b}{ }^{*} \\
\\
\quad(\mathrm{~cm})\end{array}$ & $\begin{array}{l}h_{b}^{* 3} \\
(\mathrm{~cm})\end{array}$ & $\begin{array}{l}H_{m}{ }^{*} 4 \\
(\mathrm{~cm})\end{array}$ & $\begin{array}{l}h_{m}{ }^{* 5} \\
(\mathrm{~cm})\end{array}$ & $x / h_{b}$ & $\begin{array}{l}\text { bed } \\
\text { slope }\end{array}$ & $\xi^{* 6}$ & $\begin{array}{l}\text { flume } \\
\text { type }\end{array}$ & $\begin{array}{l}\text { breaker } \\
\text { type }\end{array}$ \\
\hline 1 & 2.0 & 13.8 & 13.3 & 7.2 & 8.6 & 5.3 & $1 / 15$ & 0.45 & 1 & $\mathrm{P}$ \\
\hline 2 & 2.0 & 7.9 & 9.2 & 4.7 & 6.0 & 5.3 & $1 / 15$ & 0.59 & 1 & $\mathrm{P}$ \\
\hline 3 & 2.0 & 5.2 & 5.6 & 3.6 & 3.6 & 5.3 & $1 / 15$ & 0.73 & 1 & $\mathrm{P}$ \\
\hline 4 & 1.2 & 13.8 & 15.7 & 8.3 & 11.7 & 5.1 & $1 / 20$ & 0.20 & 1 & $\mathrm{~S}$ \\
\hline 5 & 1.2 & 12.0 & 11.3 & 7.1 & 8.4 & 5.1 & $1 / 20$ & 0.22 & 1 & $\mathrm{~S}$ \\
\hline 6 & 1.2 & 5.5 & 5.8 & 3.4 & 4.3 & 5.1 & $1 / 20$ & 0.32 & 1 & $\mathrm{~S}$ \\
\hline 7 & 1.4 & 13.6 & 17.5 & $\begin{array}{l}9.5 \\
7.4 \\
3.8\end{array}$ & $\begin{array}{l}15.5 \\
14.0 \\
10.0\end{array}$ & $\begin{array}{l}2.3 \\
4.0 \\
8.5\end{array}$ & $1 / 20$ & 0.23 & 1 & I \\
\hline 8 & 1.3 & 14.4 & 17.5 & 8.6 & 14.0 & 4.0 & $1 / 20$ & 0.21 & 2 & I \\
\hline $\begin{array}{l}{ }^{{ }^{*} 1} \mathrm{Wa} \\
{ }^{*} 2 \mathrm{Bre} \\
{ }^{* 3} \mathrm{Bre} \\
{ }^{{ }^{*} 4} \mathrm{Wa} \\
{ }^{{ }^{*}} \mathrm{Wa} \\
{ }^{*} \mathrm{Wa} \\
{ }^{*} \mathrm{Sur} \\
{ }^{*} \mathrm{Flu} \\
{ }^{*} \mathrm{P}:\end{array}$ & $\begin{array}{l}\text { peric } \\
\text { sing y } \\
\text { sing y } \\
\text { heigl } \\
\text { r dep } \\
\text { simila } \\
\text { e 1: } \text { fl }\end{array}$ & $\begin{array}{l}\text { d } \\
\text { ave heigh } \\
\text { ater deptl } \\
\text { it at meas } \\
\text { h at meas } \\
\text { rity paran } \\
\text { ume widtl } \\
\text { breaker, }\end{array}$ & $\begin{array}{l}\text { ing loc } \\
\text { ing loc } \\
\text { er } \xi= \\
\text { f } 25 \mathrm{cr} \\
\text { spillin }\end{array}$ & $\begin{array}{l}\text { ion } \\
\text { ion } \\
\text { n } \theta / \sqrt{ } \\
\text { Flume } \\
\text { breake }\end{array}$ & um & ho & ans & : off & wave & h) \\
\hline
\end{tabular}

\section{Results and Discussions}

The statistical properties of the measured instantaneous transverse velocity, $v(x, y, z, t)$, were evaluated using Reynolds decomposition based on the ensemblemean for $N=30$ trials for each case; that is, the ensemble-mean transverse velocity is defined by

$$
\overline{v(x, y, z, t)}=\frac{1}{N} \sum^{N} v(x, y, z, t),
$$

and the transverse turbulence energy

$$
q_{t}=\frac{1}{2} \overline{v^{\prime 2}}
$$

where $v^{\prime}(x, y, z, t)=v(x, y, z, t)-\overline{v(x, y, z, t)}$.

In this section, overall features of the transverse velocity distributions in the surf zone are first explained in section 3.1. Section 3.2 then describes the evolution of the length, time, and velocity scales governed by the organized flows. The transverse flow properties are parameterized in section 3.3.

\subsection{Temporal variation in transverse velocity distributions}

Fig. 4 shows the distributions of $\bar{v}$ and $q_{t}$ at the trough level in spilling (case 4) and plunging (case 1) breakers in the transition region, together with the time record of surface elevation $(\eta)$. We found in the case of spilling waves that negative 
July 29, 2015 12:11 WSPC/INSTRUCTION FILE "Characteristic time, length and velocity scales of transverse flows in the surf zone"
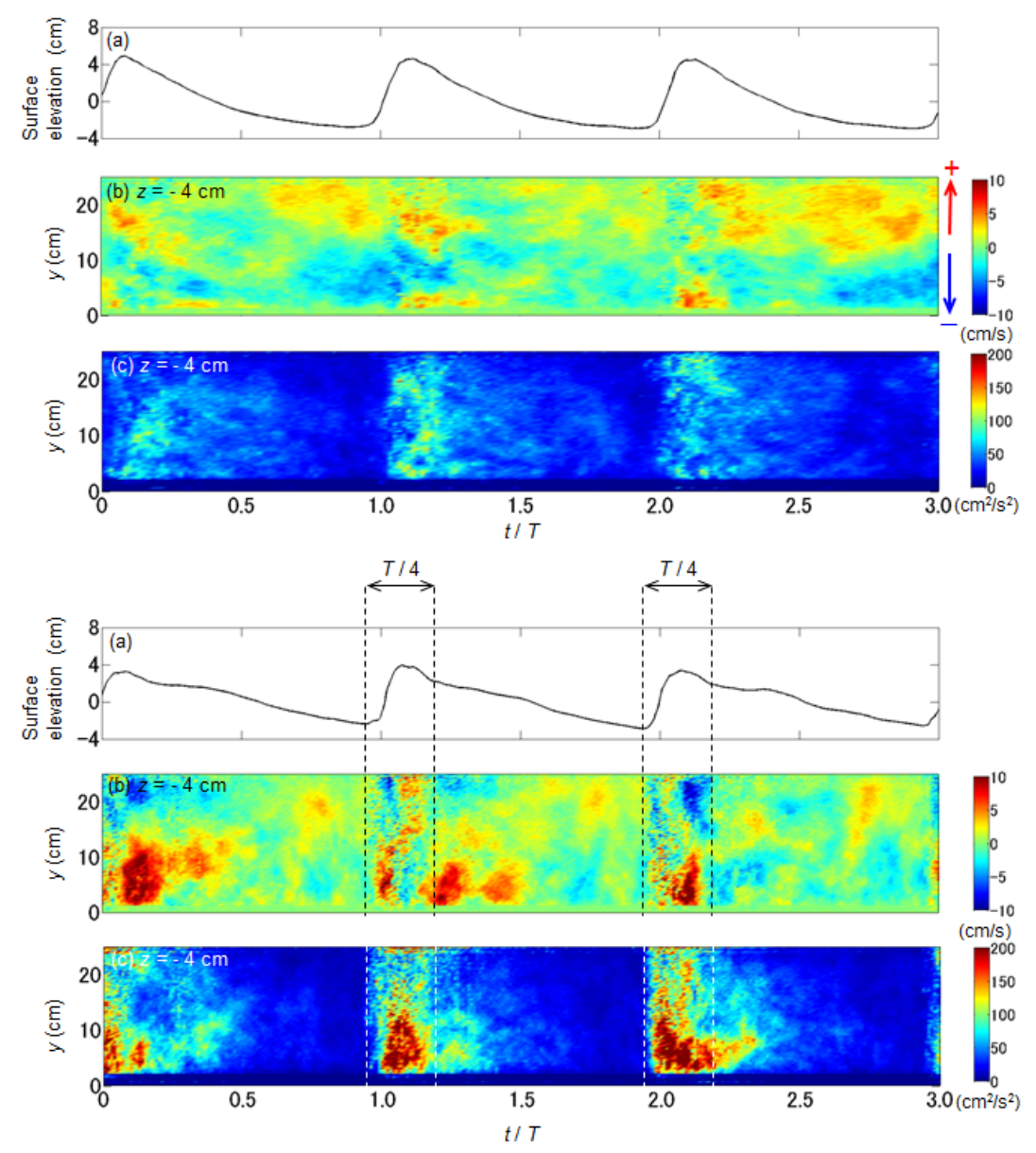

Fig. 4. Temporal variations in ensemble-averaged (a) surface elevation, and transverse distributions of (b) transverse velocity $(\bar{v})$ and (c) turbulent energy $\left(q_{t}\right)$ beneath the wave trough level $(z=-4$ $\mathrm{cm}$ ); case 4 (top panel) and case 1 (bottom panel). The arrows beside the figure (b) present example flow orientations with positive and negative velocities.

ensemble velocity appears in a half area of the flume width, $y \sim 0$ to $12 \mathrm{~cm}$, and the positive one in another side area over every wave; that is, a steady pattern of divergent flow from the flume center towards both sidewalls. Minor fluctuations of the ensemble mean flow were observed on the breaking wave face where laterally uniform distributions of the turbulent energy occur. In the plunging wave case, the transverse flow pattern changes in the orientation over the flume width several times in one wave period. In particular, rapid alternations of the convergent and divergent transverse flows were observed in the duration of $T / 4$ when significant turbulence energy appeared on the wave face. These results indicate that coherent patterns of transverse flows are formed for both breakers in the surf zone, while the amplitudes 
July 29, 2015 12:11 WSPC/INSTRUCTION FILE "Characteristic time, length and velocity scales of transverse flows in the surf zone"
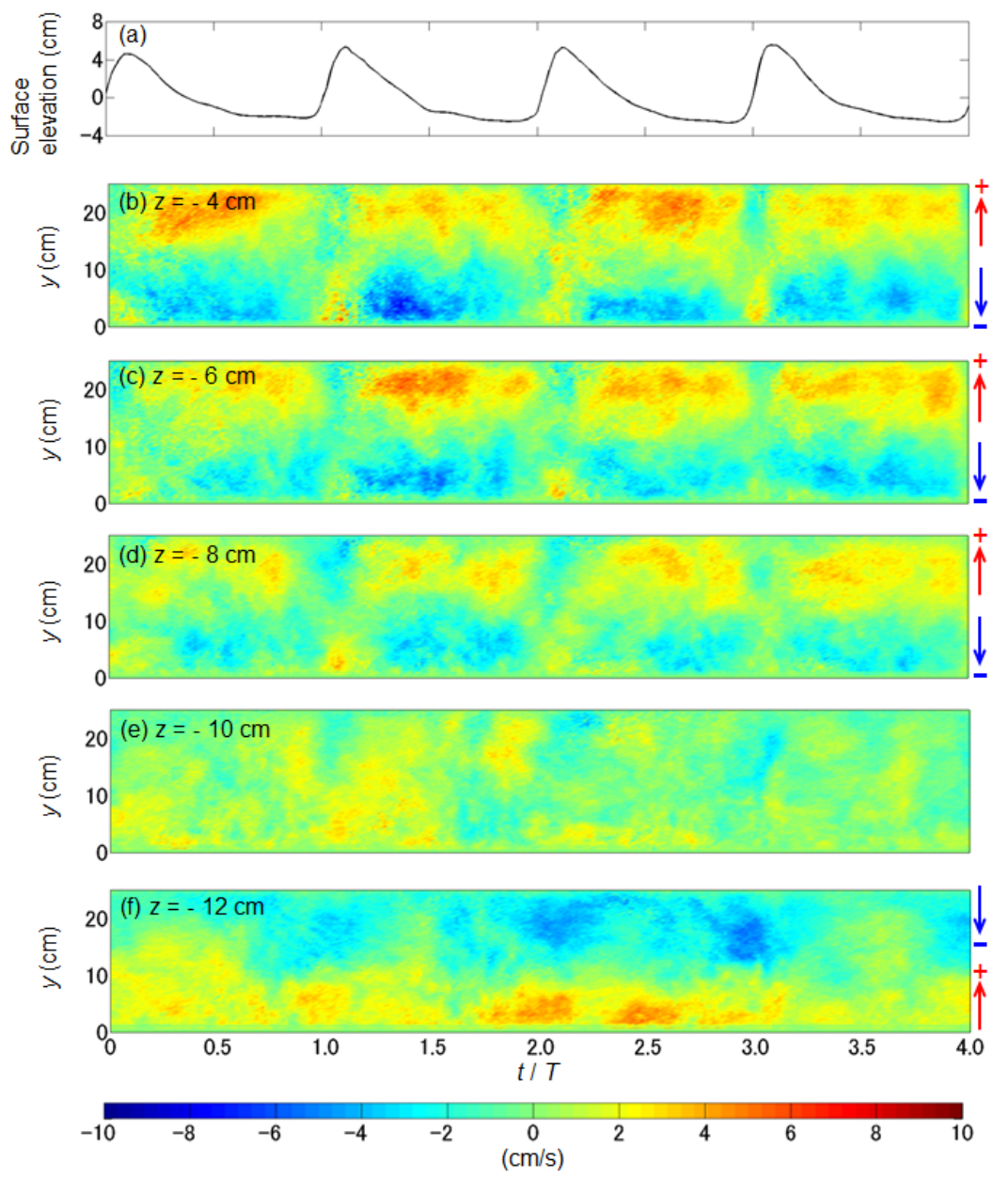

Fig. 5. Temporal variation in ensemble-averaged (a) surface elevation and (b)-(f) transverse velocity $(\bar{v})$ distributions from the trough to the bottom level in the transition region $\left(x / h_{b}=4.0\right)$ for Case 7 . The arrows beside the figures present example flow orientations with positive and negative velocities.

and frequencies of the variations of the flows are breaker-dependent. No transverse fluid motion or no lateral deformation of the wave crest was observed before wave breaking for any breaker. As we focus on the organized three-dimensional flows in this study, the transverse patterns of ensemble-mean flows are discussed in the remaining part of the paper rather than the turbulent energy with uniform statistical features in the transverse direction.

Fig. 5 shows the distributions of the ensemble-mean transverse velocity for all measurement levels $(z=-4$ to $-12 \mathrm{~cm})$ at $x / h_{b}=4.0$ for case 7 (see Table 1 ). The convergent flows emerge in the upper layer $(z=-4,-6$, and $-8 \mathrm{~cm})$ within the short duration on the front face of breaking waves, which changes in the orientation for 
July 29, 2015 12:11 WSPC/INSTRUCTION FILE "Characteristic time, length and velocity scales of transverse flows in the surf zone"

Characteristic time, length and velocity scales of transverse flows in the surf zone 9

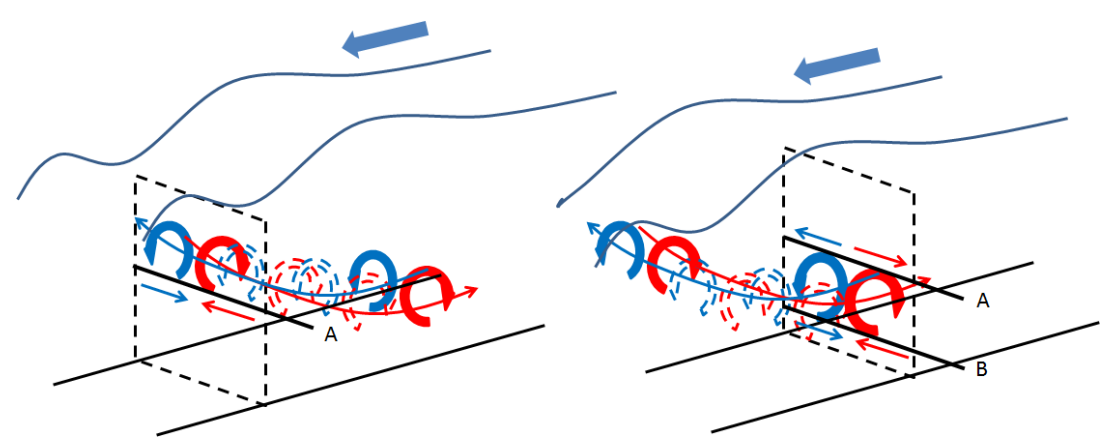

Fig. 6. Schematic illustration of the relative locations between UVP measurement line and vortex structure at the phase of wave arrival (left) and after the wave has passed (right).
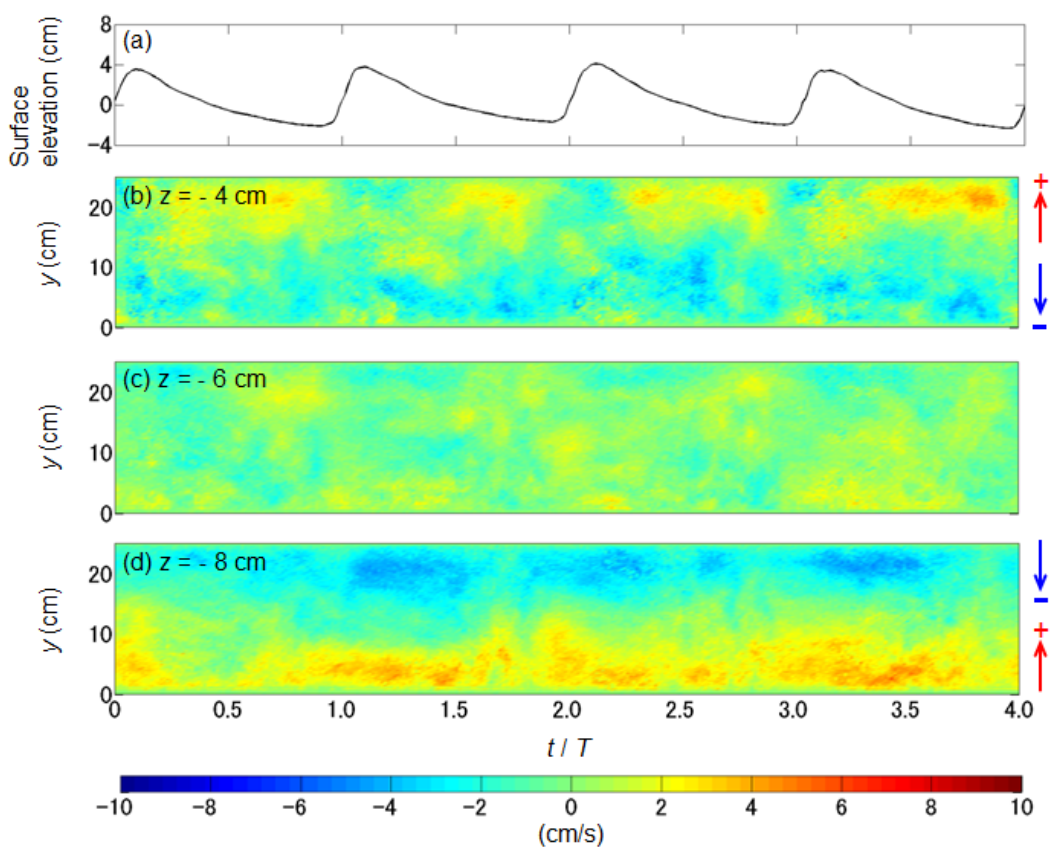

Fig. 7. Temporal variation in ensemble-averaged (a) surface elevation and (b) $-(d)$ transverse velocity $(\bar{v})$ distributions from the trough to the bottom level in the bore region $\left(x / h_{b}=8.5\right)$ for Case 7 . The arrows beside the figures present example flow orientations with positive and negative velocities.

being a quasi-steady divergent flow before the arrival of the next wave. In contrast, a steady convergent flow from both sides of the flume towards the center of the flume occurs near the bottom $(z=-12 \mathrm{~cm})$.

Similar structures of secondary rotational flows have often been observed in steady open channel flows (Rodrigez and Garcia 2008, Blanckaert 2010). The trans- 
July 29, 2015 12:11 WSPC/INSTRUCTION FILE "Characteristic time, length and velocity scales of transverse flows in the surf zone"

verse flows associated with the secondary vortices have been found to be sustained if the bed's roughness is sufficient to provide transverse oscillations in the bottom turbulent shear, regardless of the width of the channel. In the present wave experiments, we did not observe any transverse flow in pre-breaking waves or at the breaking location where there is wave-induced bottom shear comparable to that inside the surf zone. Thus, the roughness of the bed may be irrelevant to the observed flows. Watanabe and Saeki (1999) found that a transverse velocity was produced initially at the wave-plunging location when an overturning jet splashes on an undisturbed water surface. The organized patterns of the transverse flows with opposite orientations were then induced successively in a transverse array of stream-wise counter-rotating vortices formed during a splash-up cycle. The analogous features of the observed flow variations, shown in Fig. 4 and Fig. 5 (b) - (d), to the previous computed results indicate that the measured transverse flows were also associated with the counter-rotating vortices produced in experimental breaking waves (see Fig. 6). According to Watanabe et al. (2005), the computed counter-rotating vortices are wrapped by the following roller vortex and are thus stretched obliquely downwards, evolving into ODEs, organizing three-dimensional structures, which are transported towards the shore behind the wave front. Fig. 6 illustrates schematically how the measured flow orientation alternates in the vortex structure transported by breaking waves. As a pair of the stream-wise vortices emerge near the plunging wave surface and stretched obliquely downward, the convergent transverse velocity near the bottom parts of the vortex pair was measured at the transducer location ' A' below the trough level when the wave front arrives at the measurement location (see Fig. 6 left). Because the ODEs are then transported towards the shore, due to the wave-induced horizontal flows, the vortex axes intersect the measurement line over the flume span after the wave has passed, and the opposite velocity profile (divergent flow) is measured by the UVP at the fixed level ' $\mathrm{A}$ ' on the upper parts of the stretched vortices (See Fig. 6 right). The UVP transducer, installed near the flume bottom B, detects the steady convergent flows at the bottom parts of the vortices that always lie over the bottom, as shown in Fig. $5(z=-12 \mathrm{~cm})$. Because the ODEs are produced with every wave plunging during the splash-up process, repeating alternations of the flow orientation, due to the passage of multiple vortices, are observed in the plunging wave case (see Fig. 4 bottom). The frequency of the alternation of the flow orientation is reduced in the spilling breaker because of less numbers of the vortex arrays produced (see Fig. 5).

Cross-shore variations of the transverse flow are found in a comparison of Fig. 5 with Fig. 7, showing the transverse velocity in the bore region for case 7 . As fully developed turbulence containing in the bore wave disturbs the flows near the surface $(z=-4 \mathrm{~cm})$, small-scale fluctuations in the velocity continue with higher frequency variations under the bore over longer durations (about $T / 2$ ) until the divergent flow dominates. Steady convergent bottom flows were observed over the waves (see Fig. $7, z=-8 \mathrm{~cm})$. 
July 29, 2015 12:11 WSPC/INSTRUCTION FILE "Characteristic time, length and velocity scales of transverse flows in the surf zone"

Experimental evidence for organized longitudinal vortical flows formed in the laboratory surf zone has been provided in the previous discussion. However, because the flume sidewalls may be associated with the formations of the transverse flows within the flume width, the effects of the walls on the observed flows must be identified to understand invariant features of the observed flows.

Fig. 8 shows the transverse velocity in Flume 2 with a flume width wider than that of Flume 1 (see Table 1 for dimensions). We found two pairs of divergent and convergent flows regularly aligned in the transverse direction between the walls at shallower levels $(z=-4,-6$, and $-8 \mathrm{~cm})$ while transverse bottom flows with the opposite orientations to the upper flows were observed to vary in synchronization with the phase change of the upper flows (Fig. $8 z=-12 \mathrm{~cm}$ ). We may thus conclude that transverse flow patterns induced by two pairs of counter-rotating vortices were formed over the flume width in this case (see Fig. 9). The wavelengths for the transverse undulations of the flows were estimated to be in the range of $24-26$ $\mathrm{cm}$, the same as observed in Flume 1 (see Fig. 5). The agreement of the observed length-scales of the transverse flows in both flumes suggests no explicit contribution of the flume width to the formation of the organized vortices. Also, no transverse velocity was measured outside the surf zone, reinforcing the conclusion that the observed vortices resulted from the breaking process regardless of the sidewalls. However, whether once the vortices are formed at an early stage, the sidewalls may affect the later transverse growth of the vortices and thus may constrain the maximum transverse length scale of the flow through the breaking process, should be investigated in future work.

\subsection{Scaling the transverse flows}

Variations in the length and time scales of the vortex-induced flows, depending on the breakers, were investigated. Because a common Fourier spectrum for a nonperiodic finite data sample (128 data points) along the measurement line provided poor estimates of characteristic wavelengths for the observed velocity undulations, a primitive scaling definition for irregular signals in terms of a zero-up crossing method was used in the present analysis. The transverse length scale of the flows, $\lambda(t)$, was defined as the ensemble mean interval between successive zero-up crossing locations for the instantaneous velocity undulations over the transverse measurement line, $\lambda(t)=\frac{1}{N} \sum_{j}^{N}\left[\frac{1}{M_{j}} \sum_{i}^{M_{j}} \lambda_{i j}(t)\right]$ (see the example velocity plot on the B-B' cross-section line in Fig. 10). Here, $\lambda_{i j}(t)$ is the $i$ th instantaneous length interval for the $j$ th trial experiment. $M_{j}$ is the maximum number of the estimated length intervals for the $j$ trial of total $N=30$ trials for each case. The same definition was also used for determining the time scale of the flows, $\tau(t)$; the ensemble mean time interval of the successive zero-up crossing phases, $\tau_{j}(t)$, for the time record of the 
July 29, 2015 12:11 WSPC/INSTRUCTION FILE "Characteristic time, length and velocity scales of transverse flows in the surf zone"
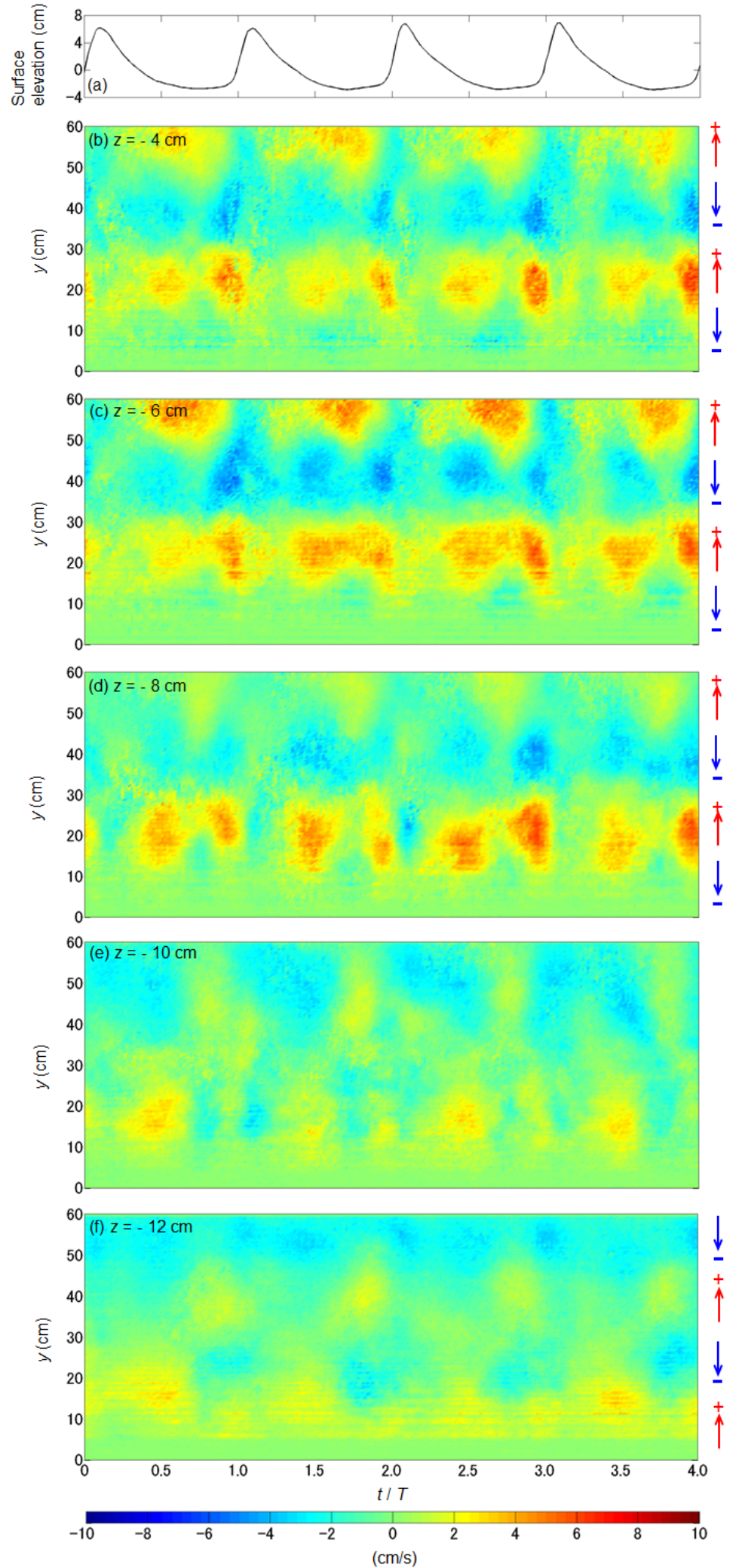

Fig. 8. Temporal variation in ensemble-averaged (a) surface elevation and (b)-(f) transverse velocity $(\bar{v})$ distributions from the trough to bottom level in the transition region $\left(x / h_{b}=4.0\right)$ for Case 8 . The arrows beside the figures present example flow orientations with positive and negative velocities. 
July 29, 2015 12:11 WSPC/INSTRUCTION FILE "Characteristic time, length and velocity scales of transverse flows in the surf zone"

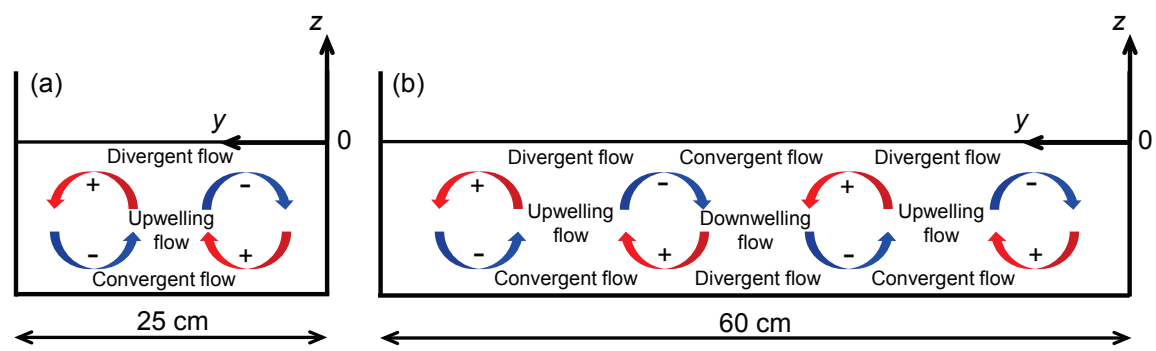

Fig. 9. Schematic diagram of transverse organization of counter-rotating vortices on the crosssections of (a) Flume 1 (Case 7) and (b) Flume 2 (Case 8).

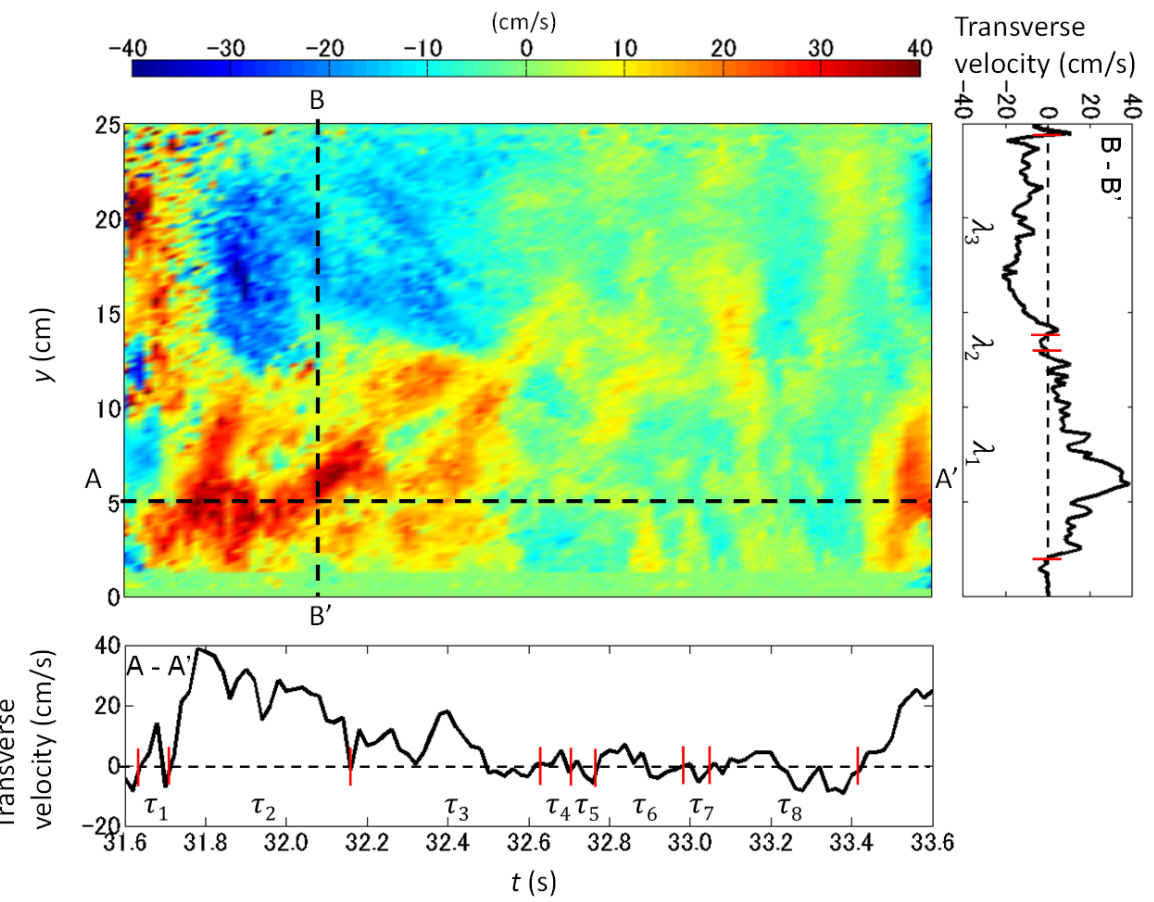

Fig. 10. Definitions of the length and time scales of the transverse flow; a velocity line plot on the B-B' cross-section (over the flume span) of the instantaneous velocity distribution (right), and a velocity line plot on the A-A' cross-section (over the time sequence) of the instantaneous velocity distribution (bottom).

instantaneous velocity, $\tau(t)=\frac{1}{N} \sum_{j}^{N} \tau_{j}(t)$ (see the velocity plot on the A-A' crosssection line) at the location where the maximum velocity is achieved ( $y \sim 5 \mathrm{~cm}$ in Flume 1). The root-mean-squares of the transverse velocity over the measurement 
July 29, 2015 12:11 WSPC/INSTRUCTION FILE "Characteristic time, length and velocity scales of transverse flows in the surf zone"

line defines the velocity scale of the flow, $\gamma(t)=\left(\frac{1}{N} \sum_{j}^{N} v_{j}(t)^{2}\right)^{1 / 2}$ (superscript indicates transverse average). Because the deviation from the transverse average of the velocity is considered to constitute the turbulent fluctuating field (Lakehal and Liovic 2011), $\gamma$ provides an appropriate measure of the transverse fluctuation velocity, assuming zero transverse average of the instantaneous flow, $\tilde{v}_{j}=0$. The dimensionless velocity, $\gamma / \sqrt{g h_{m}}$, represents relative transverse velocity with respect to the long-wave celerity; that is, the relative dominance of a gravity effect to the transverse convection. The dimensionless diffusivity, $\gamma \lambda / \nu$, indicates a ratio of transverse diffusion due to the stream-wise vortices to the viscous one $(\nu)$. In addition to $\gamma / \sqrt{g h_{m}}$ and $\gamma \lambda / \nu$, the temporal variation in the dimensionless scales of the length $(\lambda / W)$ and time $(\tau / T)$ for the phases of breaking waves in cases $1-3$ (plunging waves; see Table 1) are shown in Fig. 11. Here, $W$ is the representative transverse length, taken to be the flume width, and wave period $T$ is used as the reference time scale.

We found inherent features of time-dependent flow scales corresponding to the plunging wave phases; that is, $\lambda / W$ and $\tau / T$ decrease at the passage of each wave breaking crest while $\gamma / \sqrt{g h_{m}}$ increases rapidly at this phase, indicating that the energetic vortices induce the transverse flow with the rapid alternation of flow orientation in the short duration when the plunging wave arrives at the measurement location. The dimensional scales at this phase were estimated to be $\lambda_{\min } \sim 5 \mathrm{~cm}$, $\tau_{\min } \sim 0.2-0.3 \mathrm{~s}$ and $\gamma_{\max } \sim 20-30 \mathrm{~cm} / \mathrm{s}$, respectively. $\lambda / W$ and $\tau / T$ then increase on the rear face of the waves due to the quasi-steady divergent flows over the flume width, as appeared in Fig. 5, while $\gamma / \sqrt{g h_{m}}$ attenuates with correlation to the surface elevation. The relative diffusivity, $\gamma \lambda / \nu$, exhibits significant differences in the amplitude and the duration between cases 1 to 3 as nonlinear amplifications between $\lambda$ and $\gamma$ enhance local convections in the vortex structure discussed in the previous section (see also Fig. 9).

In spilling breakers (cases 4 to 6 ), longer mean time scales and lower velocity scales than those in the plunging cases were observed (see Fig. 12). The length scales and the relative diffusivity show unorganized fluctuations with less amplitude than the plunging ones, indicating quasi-steady transverse diffusion process in the spilling waves.

The cross-shore evolution of the estimated scales at the trough level for case 7 is shown in Fig. 13. The time records for the plunging location $\left(x / h_{b}=2.3\right)$ present the longest mean length, and time scales and the lowest velocity and diffusivity with less amplitudes of the variations, compared with the other locations. With wave propagation, the typical in-phase scale variations of $\lambda / W, \tau / T$, and $\gamma \lambda / \nu$ are amplified, and the relative velocity increases as fluid convections by the vortices are intensified in the breaking process (see also Fig. 5$)$. In the bore region $\left(x / h_{b}=8.5\right)$, the inphase variations of the length and time scales occur prior to the maximum surface 
July 29, 2015 12:11 WSPC/INSTRUCTION FILE "Characteristic time, length and velocity scales of transverse flows in the surf zone"
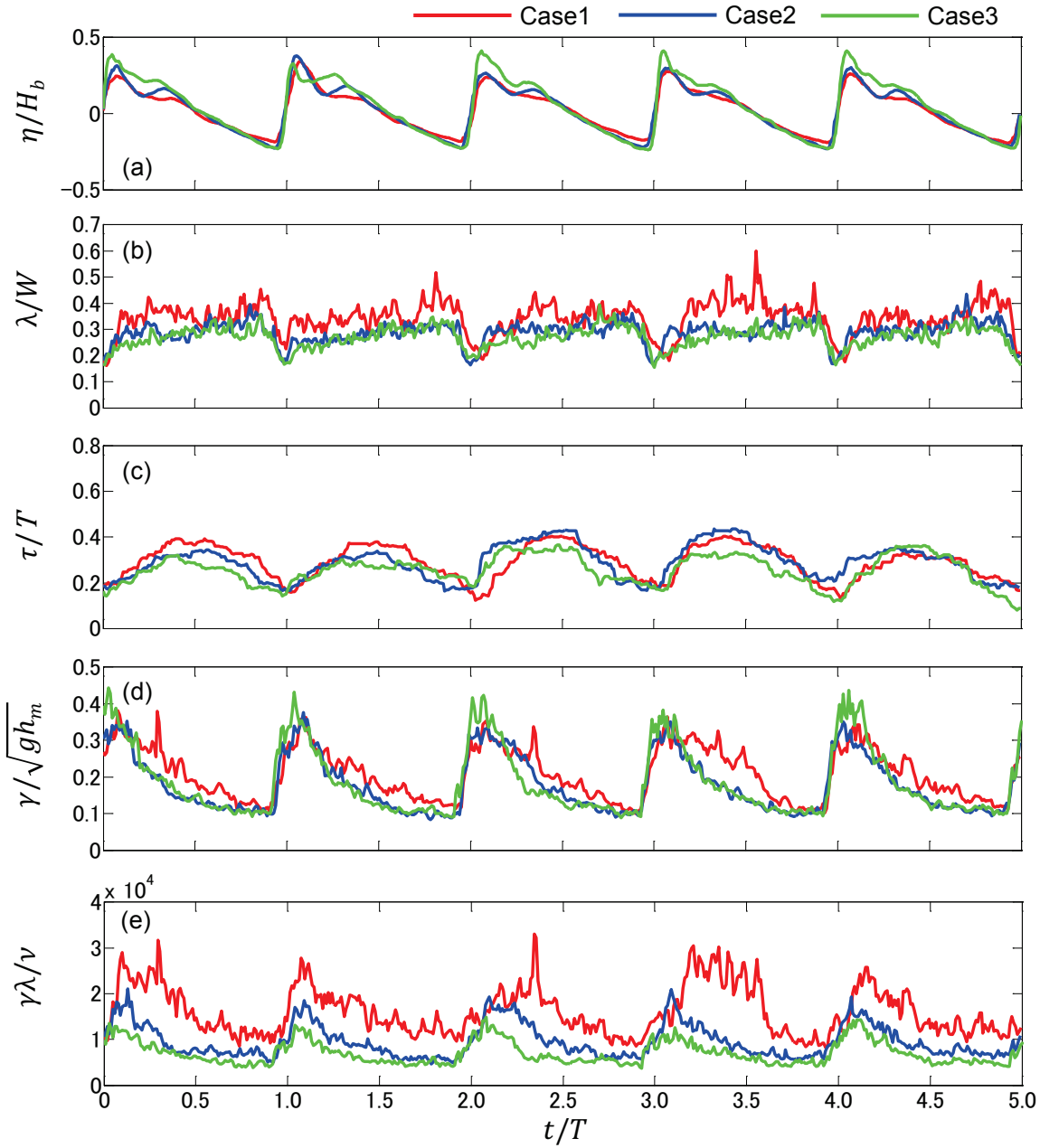

Fig. 11. Time records of the dimensionless surface elevation, length, time, and velocity scales of the transverse flow at $z=-4 \mathrm{~cm}$ (trough level) for plunging waves (red line; Case 1, blue line; Case 2, green line; Case 3).

elevation phase, as the preceding turbulent bore explicitly enhances alternations of the flow orientation (see also Fig. 7).

\subsection{Parameters characterizing flow scales}

The length and time scales take the minimum values, $\lambda_{\min }$ and $\tau_{\min }$, on the breaking wave face, and then increase to take the maximum values, $\lambda_{\max }$ and $\tau_{\max }$, on the rear face in the in-phase variations as already discussed. Contrary, the maximum velocity, $\gamma_{\max }$, is achieved on the wave face. These maximum and minimum scales averaged over waves for all trials, $\overline{\lambda_{\max }}, \overline{\lambda_{\min }}, \overline{\tau_{\max }}, \overline{\tau_{\min }}$, and $\overline{\gamma_{\max }}$, are defined as 
July 29, 2015 12:11 WSPC/INSTRUCTION FILE "Characteristic time, length and velocity scales of transverse flows in the surf zone"
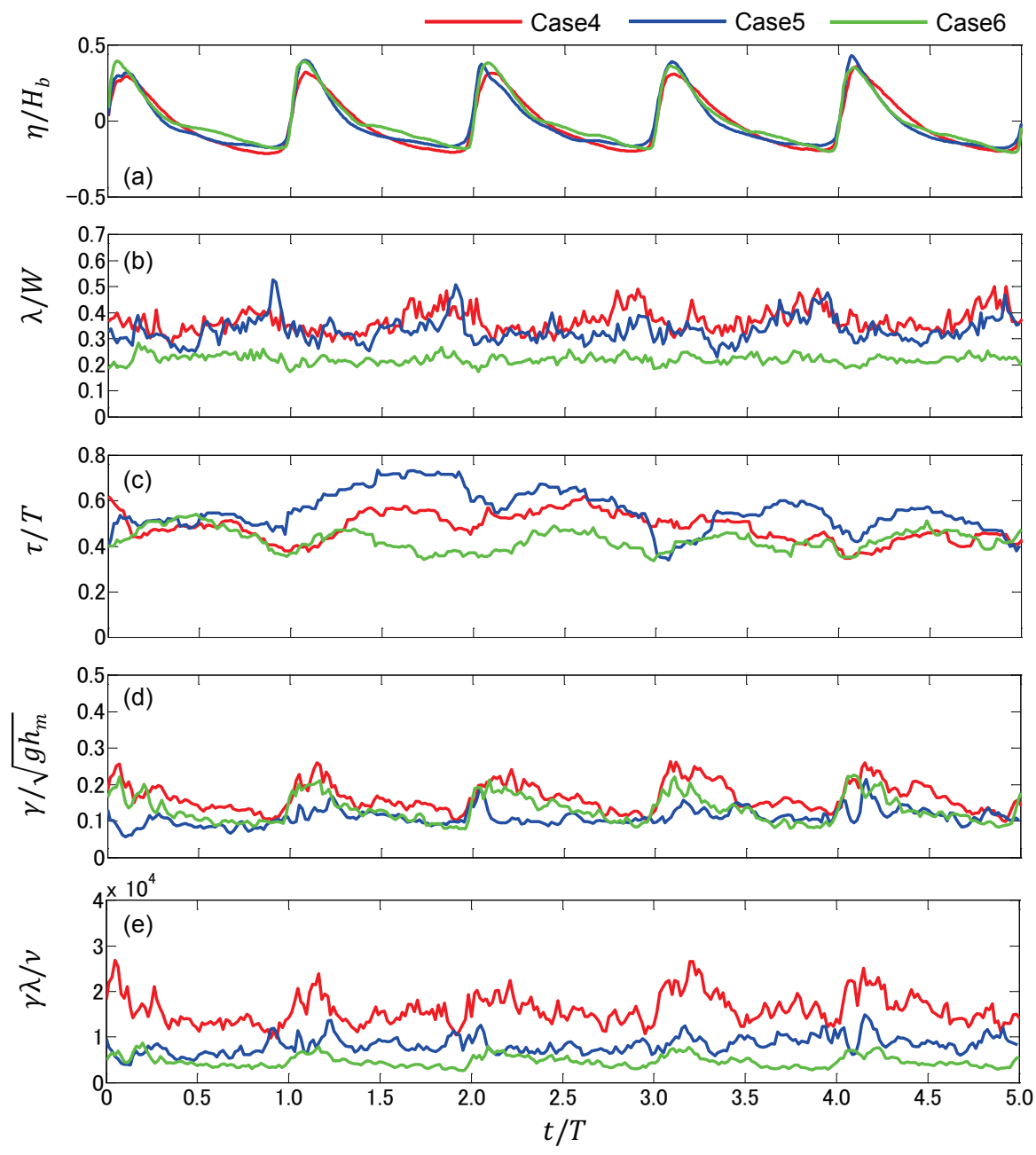

Fig. 12. Time records of the dimensionless surface elevation, length, time and velocity scales of the transverse flow at $z=-4 \mathrm{~cm}$ (trough level) for spilling waves (red line; Case 4, blue line; Case 5, green line; Case 6).

the characteristic scales for the in-phase variations of the transverse flow, which are parameterized for case 1 through 7 .

The mean length scales, $\overline{\lambda_{\max }} / W$ and $\overline{\lambda_{\min }} / W$ as a function of transverse Froude number defined by $\mathrm{Fr}=\overline{\gamma_{\max }} / \sqrt{g h_{m}}$ are shown in Fig. 14 (left). The linear decrease in the maximum and minimum lengths with dispersions with respect to $F r$ indicates that the transverse intervals where the stream-wise vortices are produced shorten with an increase in the transverse flow velocity. We also found monotonic decreases of both mean time scales, $\overline{\tau_{\max }} / T$ and $\overline{\tau_{\min }} / T$, with $F r$, supporting the observed frequency increase of the alternation of the flow direction with the flow velocity, as 
July 29, 2015 12:11 WSPC/INSTRUCTION FILE "Characteristic time, length and velocity scales of transverse flows in the surf zone"
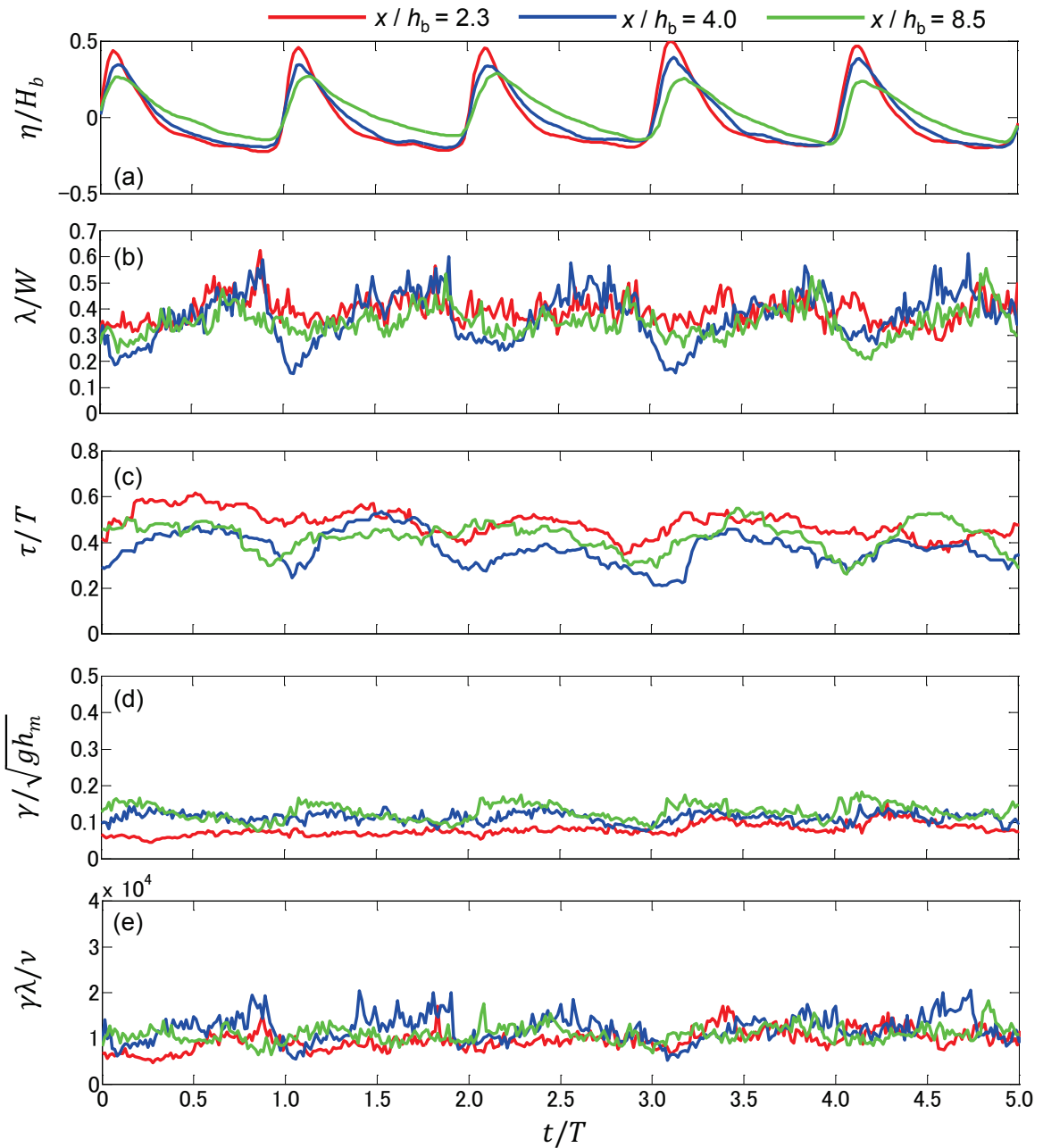

Fig. 13. Time records of the dimensionless surface elevation, length, time, and velocity scales of the transverse flow at $z=-4 \mathrm{~cm}$ (trough level) for the intermediate breaker (Case 7) at three cross-shore locations $x / h_{b}=2.3$ (plunging region), at $x / h_{b}=4.0$ and at $x / h_{b}=8.5$ (bore region).

discussed in section 3.2

Fig. 15 shows the relation between the Froude number in the transition region and the surf similarity parameter, $\xi=\tan \theta / \sqrt{H_{b} / L_{0}}$, together with the fitted empirical line.

We found a linear correlation of $F r$ with $\xi$, indicating a breaker dependency of the transverse velocity; that is, with increase of $\xi$, the transverse convention is relatively intensified with respect to the gravity wave motion.

The empirical linear relations of the length and time in terms of $\mathrm{Fr}$, shown in Fig. 14, and the correlation between $F r$ and $\xi$ (Fig. 15) provide explicit breaker 
July 29, 2015 12:11 WSPC/INSTRUCTION FILE "Characteristic time, length and velocity scales of transverse flows in the surf zone"
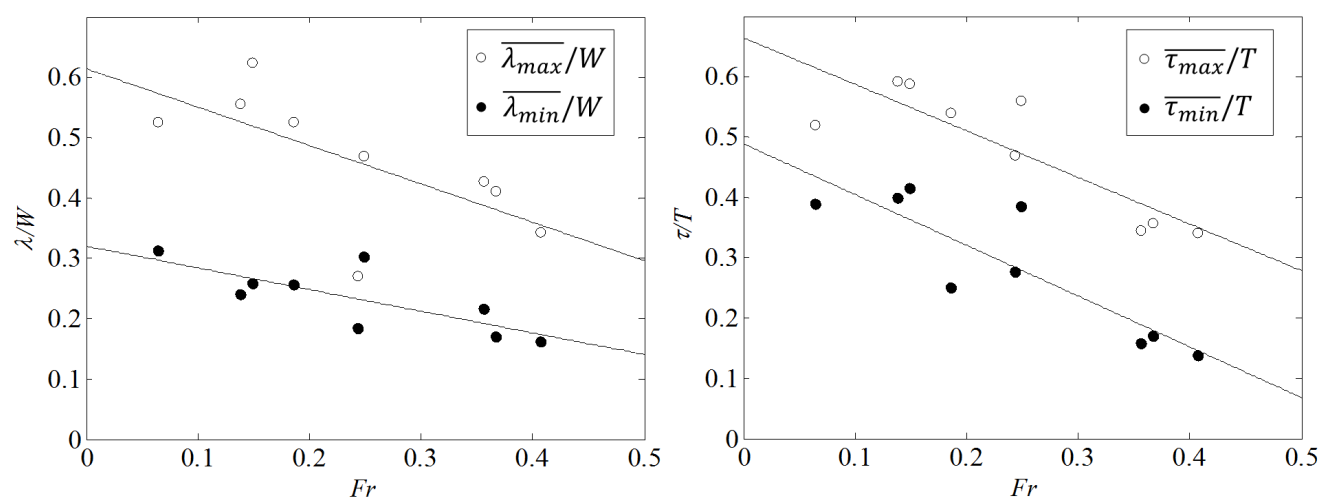

Fig. 14. Relationship between the Froude number and the length (left) and time (right) scales of the transverse velocity with empirical fitted lines of $\overline{\lambda_{\max }} / W \approx-0.64 F_{r}+0.61, \overline{\lambda_{\min }} / W \approx$ $-0.36 F_{r}+0.32, \overline{\tau_{\max }} / T \approx-0.77 F_{r}+0.66$ and $\overline{\tau_{\max }} / T \approx-0.84 F_{r}+0.49$.

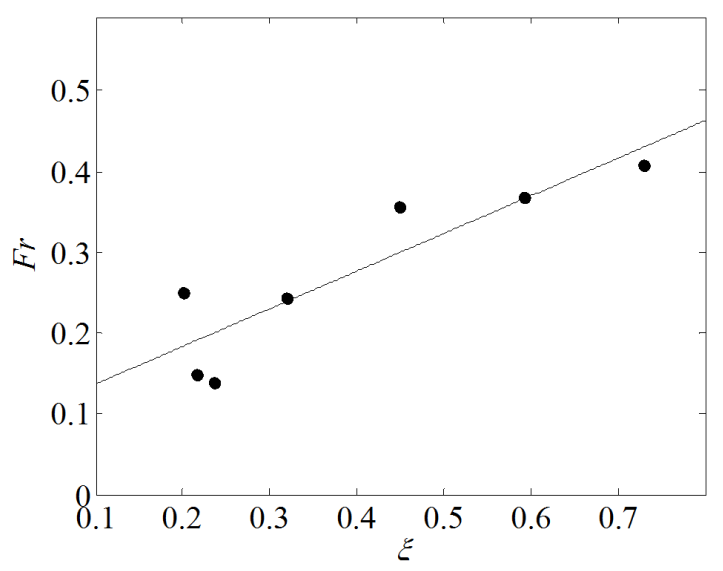

Fig. 15. Relationship between the Froude numbers for the transverse flows in the transition region and the surf similarity parameters with the empirical fitted line of $F r \approx 0.47 \xi+0.09$.

dependencies of the length and time scales on $\xi$ (see Fig. 16).

We found that the length scales with dispersions may be described by the empirical linear approximation with the similarity parameter (Fig. 16 left). On the other hand, while linear decreases of both time scales were observed in the spilling breaking regime $(\xi<0.4$, see Batjjes 1974), a constant value of $\tau / T \approx 0.25$ is achieved in the plunging regime. These features of the breaker-dependent scales reasonably interpret the variation in transverse flows discussed in section 3.1; that is, the energetic transverse flow with shorter transverse fluctuations change rapidly in the flow orientation in the duration of $T / 4$ when plunging waves arrive at the measurement 
July 29, 2015 12:11 WSPC/INSTRUCTION FILE "Characteristic time, length and velocity scales of transverse flows in the surf zone"
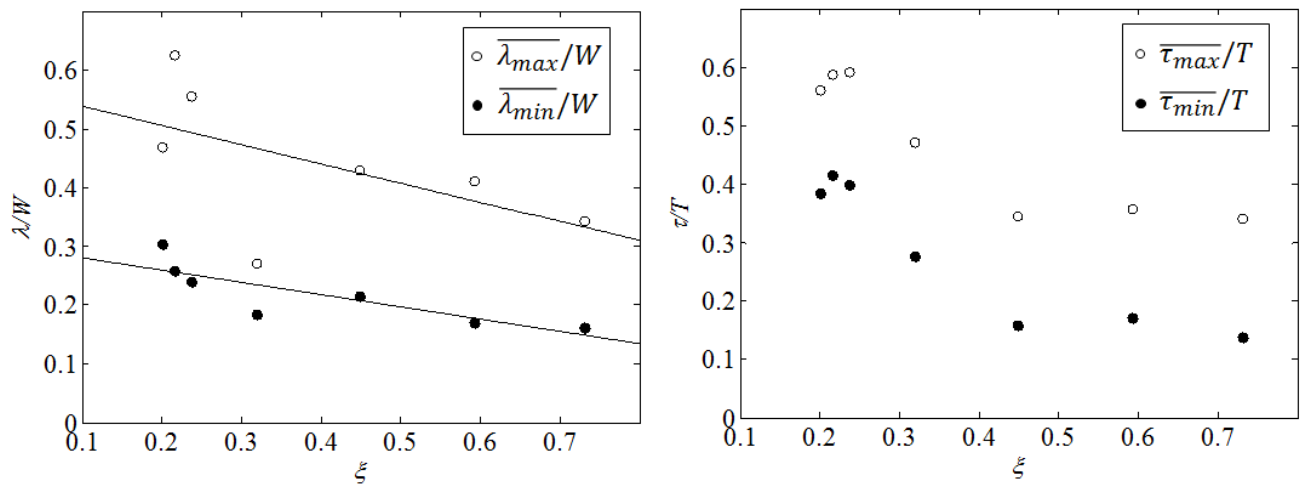

Fig. 16. Relationship between the surf similarity parameters and the length (left) and time (right) scales of the transverse velocity in the transition region with empirical fitted lines of $\overline{\lambda_{\max }} / W \approx$ $-0.33 F_{r}+0.57$ and $\overline{\lambda_{\min }} / W \approx-0.21 F_{r}+0.30$.

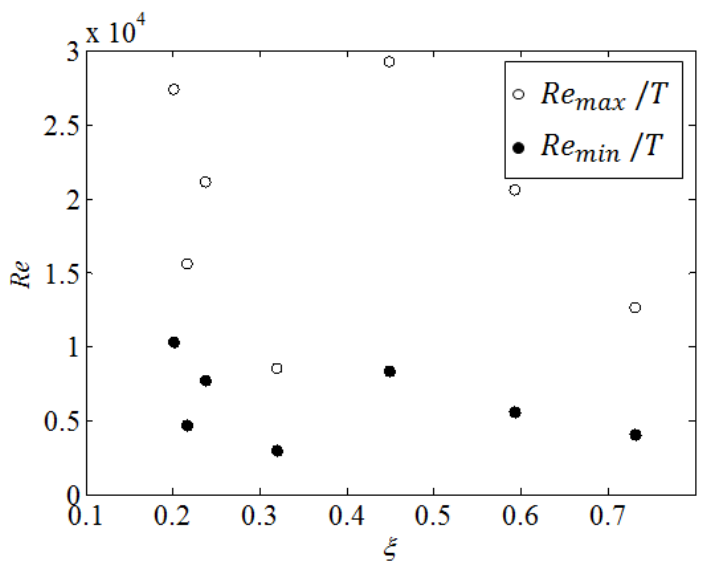

Fig. 17. Reynolds numbers for the transverse flows as a function of the relative water depth.

location (see Fig. 4 bottom) while the weaker divergent flow over the flume width changes slowly in orientation for the spilling breaker (see Fig. 4 top).

These correlations of the length, time, and velocity scales with $F r$, as well as $\xi$, indicate that the statistical features of the transverse fluid motions, due to the stream-wise vortices, are relevant to the gravity effect in the early stages of wave breaking. Because the initial formation of the horizontal roller causes the strong shear to destabilize the vorticity in the span-wise direction and thus induce streamwise vortices (Watanabe et al. 2005), the intensity of the roller vortex, governed by projection of the overturning wave crest due to gravity, consequently determines the fluid shear strength to induce the transverse fluid flows. 
July 29, 2015 12:11 WSPC/INSTRUCTION FILE "Characteristic time, length and velocity scales of transverse flows in the surf zone"

It is interesting to discuss breaker dependencies of the transverse diffusivities, featuring the in-phase variations from the minimum to maximum values during the wave passage, observed in Fig. 11(e) and Fig. 12(e). Fig. 17 shows the relationship of the maximum and minimum Reynolds numbers, $R e_{\max }=\overline{(\gamma \lambda)_{\max }} / \nu$ and $R e_{\min }=\overline{(\gamma \lambda)_{\min }} / \nu$ (or mean relative transverse diffusivities) and the similarity parameter. Unlike the other scales, no correlation was observed for Re with $\xi$. Because the transverse diffusion is induced by local shear and thus develops during the breaking process, there may be no explicit dependency on the initial gravity effects of wave motion. As further investigations are needed to identify the three-dimensional components of the fluid velocity, parameterization of the diffusion process remains for future research.

\section{Conclusions}

Distributions of transverse fluid flows were measured experimentally over a span of laboratory wave flumes using an ultrasonic velocity profiler (UVP) method. The observed mean transverse velocity changed sequentially in orientation after the breaking wave passed the measurement location; divergent and convergent flows appeared, in turn, under the breaking waves, which is consistent with the transverse flow patterns computed by Watanabe and Saeki (1999). Analogous to previous results, we conclude that the same mechanism forms the organization of the transverse flows; that is, the observed flows are driven by pairs of stream-wise counter-rotating vortices and changes in the flow orientation within the three-dimensional vortex structures evolve during the wave-breaking process.

Fundamental features of spatial and temporal variations of the transverse flows in the surf zone are discussed in terms of length and time scales defined by zeroup-crossing intervals for time records of the instantaneous velocity distributions as well as a transverse velocity scale defined by root-mean-square velocity. We found inherent features of time-dependent flow scales corresponding to the plunging wave phases. The dimensionless length and time scales decrease at the passage of each wave breaking crest, while the velocity scales increase rapidly at this phase, indicating that the energetic vortices induce the transverse flow with a rapid alternation of the flow orientation in the short duration when the plunging wave arrives at the measurement location. The transverse Froude number, defined as the ratio of the velocity scale to long-wave celerity (or relative transverse convection with respect to gravity) in a transition region, was found to correlate with the surf similarity parameter. The dimensionless length and time scales were also described empirically by a linear relationship with both the Froude number and the surf similarity parameter, indicating the explicit dependencies of the transverse flows on the breaker type or gravity-driven flows at the initial stage of wave breaking. Consequently, these findings are helpful in interpreting the mechanical contributions of the gravity effects on the shear field, resulting in organized transverse flows. The free-surface flows induced by overturning jets, due to gravity, produce a primary horizontal 
July 29, 2015 12:11 WSPC/INSTRUCTION FILE "Characteristic time, length and velocity scales of transverse flows in the surf zone"

roller after wave plunging. Violent wave splash at a higher surf similarity parameter results in higher span-wise vorticity in the roller and intensifies shear at the plunging location. Because the vorticity field is highly stretched and destabilized at a stagnation point, flow undulations emerged between the secondary jet and the roller vortex to generate a lateral array of counter-rotating vortices (Watanabe et al. 2005). The intensifications of the span-wise vorticity and shear at the stagnation region, caused by violent breakers at higher surf similarity parameters (i.e., gravity effect), enhance the unstable behaviors of the vorticities for forming pairs of energetic counter-rotating vortices inducing significant transverse flows. Transverse diffusion is not uniquely determined by either the Froude number or the surf similarity parameter because the complex evolution of the vortex structures in highly shearing flows may predominantly characterize the diffusion process rather than the initial splashing dynamics due to the gravity effect.

It should be noted that distributions of all three velocity components are required to precisely identify the three-dimensional flow structures produced in the surf zone while the current experiments acquire only transverse velocity profiles representing transverse organizations of the flows. In particular, as offshore-ward horizontal current, undertow, intensifies the local shear on the plunging location, the transverse flow structures may be modified by the current. Therefore the observed ensemble statistics presented in this paper were implicitly influenced by the time-averaged flow component including the undertow.

\section{Acknowledgments}

This research was supported by a JSPS Grant-in-Aid for Scientific Research (24360196).

\section{References}

Battjes, J.A. (1974), Surf Similarity, Proc. 14th Coastal Eng. Conf., $466-480$.

Blanckaert, K. (2010), Topographic steering, flow recirculation, velocity redistribution, and bed topography in sharp meander bends, Water Resour Res., 46, W09506, doi: 10.1029/2009WR008303.

Cox, D.T. and Anderson, S.L. 2001 Statics of intermittent surf zone turbulence and observation of large eddies using PIV Coastal Eng. J. 43, $121-131$

Christensen, E.D. and Deigaard, R. 2001 Large eddy simulation of breaking waves Coastal Eng. 42, $53-86$

Kubo H. and T. Sunamura . 2001. Large-scale turbulence to facilitate sediment motion under spilling breakers, Proc. Coastal Dynamics 2001, 212 - 221.

Lakehal, D., and P. Liovic (2011), Turbulence structure and interaction with steep breaking waves, J. Fluid Mech., 674, 522-577.

Longo, S., M. Petti, and I. J. Losada (2002), Turbulence in the swash and surf zones: a review, Coastal Eng., 45, 129-147.

Longo, S., D. Liang, L. Chiapponi, and L. A. Jimenez (2012), Turbulent flow structure in experimental laboratory wind-generated gravity waves, Coastal Eng., 64, 1-15.

Nadaoka, K., Hino, M. and Koyano, Y. 1989 Structure of the turbulent flow field under breaking waves in the surf zone J. Fluid Mech. 204, 359 - 387 
July 29, 2015 12:11 WSPC/INSTRUCTION FILE "Characteristic time,

length and velocity scales of transverse flows in the surf zone"

22 Junichi Otsuka and Yasunori Watanabe

Rodrigez, J. F. and M. H. Garcia (2008), Laboratory measurements of 3-D flow patterns and turbulence in straight open channnel with rough bed, J. Hydraulic Res., 46, 454-465.

Takeda, Y. (1995), Velocity profile measurement by ultrasonic Doppler method, Experimental Thermal and Fluid Science, 10, 444-453.

Ting, F.C.K. (2006), Large-scale turbulence under a solitary wave, Coastal Eng., 53, 441-462.

Ting, F.C.K. (2008), Large-scale turbulence under a solitary wave: Part 2, Coastal Eng., 55, 522-536.

Watanabe, Y. and Saeki, H., 1999. Three-dimensional large eddy simulation of breaking waves. Coast. Eng. Journal, vol. 41, pp. 281-301.

Watanabe, Y., Saeki, H. and Hosking, R. J., 2005. Three-dimensional vortex structures under breaking waves. J. Fluid Mech., vol. 545, pp. 291-328.

Watanabe, Y. and Mori, N., 2008. Infrared measurements of surface renewal and subsurface vortices in nearshore breaking waves. J. Geophys. Res., vol. 113, C07015, doi:10.1029/2006JC003950. 